Short report

\title{
Temporal course of the threshold and size of the receptive field of the Babinski sign
}

\author{
BRUNO ESTAÑOL \\ From the Division of Internal Medicine, Hospital General, Centro Médico Nacional, Cuauhtémoc, Mexico
}

SUMMARY The changes of threshold and receptive field of the Babinski reflex, with time, were assessed in ten patients with acute cerebrovascular lesions. It was found that the threshold of the reflex decreased gradually and the size of the cutaneous afferent reflex field enlarged with the passage of time. These findings suggest that there is a gradual release or organisation of the reflex flexor synergy of which the Babinski sign is a modified part.

Although the Babinski reflex has been carefully investigated, little attention has been paid to the temporal course of changes in threshold of the response and the size of its afferent reflex field. It has been known for a long time ${ }^{2}$ that, following an acute lesion in the cerebral hemispheres, there is initially a profound inhibition of the monosynaptic stretch reflexes and, after a variable period of time, the myotatic reflexes become hyperactive. We thought that a similar temporal course might be observed with the Babinski reflex. We postulated that (1) the threshold of the Babinski reflex must be greater at the outset of lesion and would gradually decrease with time and (2) the size of the afferent reflex field (the area from which the reflex can be elicited) should increase with time, being restricted at the inception of the injury to the S1 segment and gradually impinging on neighbouring dermatomes.

\section{Patients and methods}

We examined twenty patients with unilateral chronic cerebrovascular occlusive lesions. The patients were selected solely on the basis of myotatic hyper-reflexia and were examined for the presence of a Babinski sign elicited according to a scale of stimuli as follows:

++++ No Babinski sign even with very forceful stroking of the lateral part of the foot.

$++\quad$ Babinski sign elicited only with very forceful stroking of the lateral part of the foot.

$++\quad$ Babinski sign induced with moderately intense stroking of the external border of the foot.

Address for reprint requests: Dr Bruno Estañol, Hospital General, Centro Médico Nacional, Cuauhtémoc, 330, CP 067207, Mexico 7, DF.

Received 19 December 1982 and in revised form 1 May 1983. Accepted 31 May 1983

\section{$+\quad$ Babinski sign produced by light stroking of the lateral border of the foot. \\ 0 The sign is induced by tactile (non- nociceptive) stimulus.}

All patients were examined in the supine position. Stimulation was performed with a wooden stick. The stretch reflexes were recorded as follows: ++++ the most exaggerated response, 0 for no response and,+++++ and + for intermediate responses. We compared the intensity of the stimuli to elicit the Babinski sign (threshold) to the magnitude of the myotatic reflexes.

Ten patients with acute cerebrovascular occlusions were then examined. The patients were initially seen during the first twenty-four hours of the onset of the ictus. They were examined daily for seven days and again after two weeks, with regard to stretch reflexes of the ankles and knees, presence or absence of a Babinski sign and threshold of the Babinski sign. The strength of the dorsiflexion of the great toe and ankle was also assessed. Signs indicating the presence of a flexor reflex synergy (contraction of the quadriceps, tensor fasciae latae, etc) were also looked for.

The size of the afferent reflex field was evaluated according to the site on the lower extremity from which a Babinski sign could be elicited. The size was arbitrarily assessed as follows: 1-S1 dermatome only, 2-Entire sole of the foot, 3-Up to the ankle, 4-Up to the middle of the leg, 5-Up to the knee, 6-Up to the thigh.

The changes in the size of the afferent field were followed daily during the first seven days of the stroke and again after two weeks. The stimuli used were stroking, pinching or pricking the skin.

\section{Results}

Of the first group, 17 of 20 patients with a cerebral infarct and with myotatic hyperreflexia had a Babinski sign, and 15 patients had a Babinski reflex elicited with a very low threshold. Two patients had a Babinski sign only with very forceful stroking of 


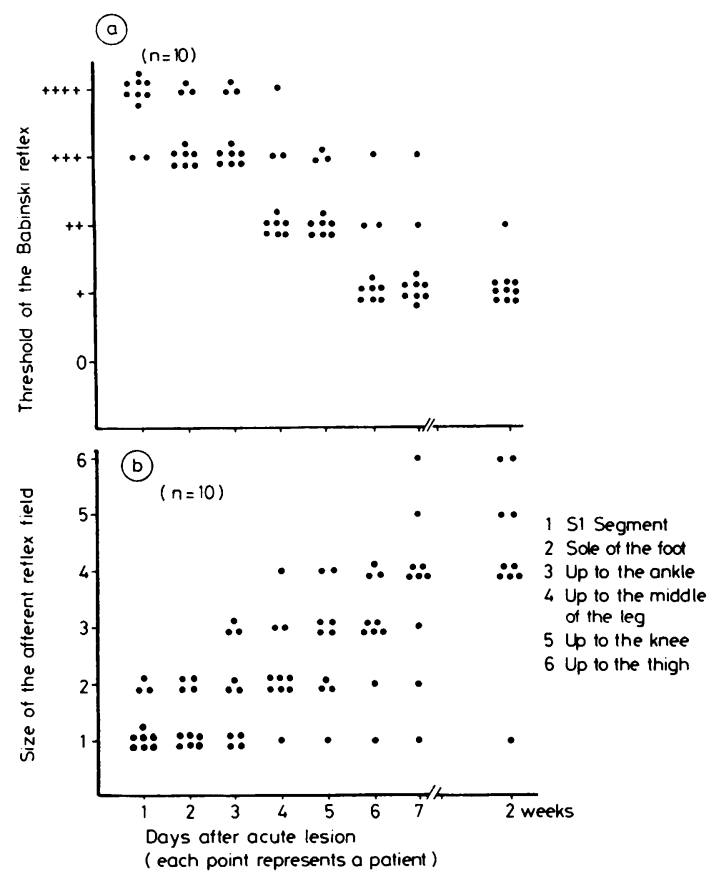

Fig (A) The threshold of the Babinski reflex decreased significantly two weeks after the inception of the ictus, (B) The size of the afferent reflex field enlarged significantly over the days after the onset of the stroke.

the sole of the foot. Three patients had no Babinski sign even with very strong nociceptive stimulation.

In the second group, after two weeks, eight of $\mathbf{1 0}$ cases had developed myotatic hyperreflexia; a Babinski sign was present in all 10. Two patients had a Babinski sign without hyperactivity of the stretch reflexes.

Correlation to the intensity of the stimulis necessary to elicit the Babinski reflex with the time elapsed since the ictus showed that the threshold gradually decreased (fig A). At the time of the ictus, the intensity of the necessary stimulus was much greater than after two weeks. At the outset of the lesion only two patients had a Babinski sign; and in these the reflex was elicited only with very forceful stimuli. Eight patients had no Babinski sign during the first twenty-four hours of the cerebral infarct. After two weeks all of the patients had developed a Babinski reflex. Nine had the response only with light stroking of the lateral part of the foot and one with moderately intense stroking.

The size of the reflex field from which the Babinski sign could be evoked increased in nine out of 10 patients. In two patients the response could be elicited by nociceptive stimuli as high as the thigh, in two patients with stimuli as high as the knee. Five patients had the Babinski sign induced by stimuli up to the middle of the leg. One patient had a Babinski sign induced only by stimulation of the S1 dermatome (fig B).

All of the patients had marked to moderate weakness of ankle and hallux dorsiflexion quantitated according to the scale of Van Gijn. ${ }^{1}$ At the end of two weeks, all of the patients had evidence of the presence of a flexor reflex synergy as manifested by various degrees of contraction of the quadriceps or tensor fasciae latae.

\section{Discussion}

It is widely accepted that the Babinski sign is a modified part of the generalised flexor reflex synergy. ${ }^{369}$ This synergy is released by the lesion of descending supraspinal pathways that project onto the interneuronal zone of the lumbosacral cord. ${ }^{1}$ At the same time there is also a lesion or functional deficit of the fibres that project directly onto the motoneurons of the effector muscles ${ }^{813}$ manifested by weakness of the dorsiflexors of the hallux, a fact already observed by Babinski in his original description. ${ }^{5}$ These two basic defects are present in most patients who display a Babinski sign.'

It has also been shown' ${ }^{9}$ that nociptive stimulation of the sole of the foot in healthy subjects results in plantar flexion of the hallux whereas stimulation of the ball of the great toe induces its dorsiflexion. This indicates that the response is a natural withdrawal from the offending stimulus. In patients with cerebral lesions the local physiological extensor reflexes are lost as their field of excitation is overwhelmed by the hyperactive flexor responses. On the other hand, Brain and Wilkinson ${ }^{7}$ demonstrated that in the newborn infant the size of the reflex field of the Babinski sign may be as high as the abdomen ipsilateral to the hallux. As the infant grows the size of the field of excitation of the reflex gradually decreases finally being confined to the ball of the great toe in the normal adult. This study suggests that in patients with cerebral lesions, the size of the field from which the abnormal reflex can be evoked enlarges to a size present in ontogenetically previous stages and furthermore that it is a dynamic process. Future studies may determine the prognostic significance of the enlargement of the size of the reflex field. Likewise, the decreasing threshold of the response must be related to the gradual emergence of the reflex flexor synergy. Unilateral acute lesions of the pyramid in rhesus monkeys ${ }^{10}$ induces hypotonicity of the muscles with sluggish myotatic reflexes and absence of a pathological extensor plantar response. After several days the stretch reflexes become hyperactive 
and a Babinski sign is easily obtained. This suggests a temporal evolution of the threshold of the monosynaptic and polysynaptic reflexes after a pyramidal tract lesion. It has also been shown ${ }^{11}$ that during the acute phase of hemiplegia, the monosynaptic stretch reflexes are severely depressed and after a variable period of time they become hyperactive, again suggesting a dynamic change in the threshold of the response.

It has been shown that an early appearance of hyperactive stretch reflexes is a good prognostic sign for the recovery of hemiplegia in man. ${ }^{11}$ It is, nevertheless, important to point out that the release of the monosynaptic stretch reflexes probably has a different mechanism from the release of the polysynaptic flexor responses; most clinicians are aware that myotatic hyper-reflexia may not be associated with a Babinski reflex and vice-versa. Van Gijn ${ }^{1}$ found that $74 \%$ of the patients with a pathological extensor reflexes had hyperactive tendon jerks. However, the two reflexes are frequently released together. It remains to be established whether the size of the field from which the response can be evoked and the threshold of the reflex stabilise after a lapse of time or if there is a gradual return to normal conditions.

\section{References}

' Van Gijn J. The Babinski sign and the pyramidal syndrome. J Neurol, Neurosurg Psychiatry 1978;41:865-73.

${ }^{2}$ Fulton JF, Kennard M. A study of flaccid and spastic paralyses produced by lesions of the cerebral cortex in primates. Res Publ Assoc Res Nerv Ment Dis 1934;13:158-210.

${ }^{3}$ Walshe FMR. The plysiological significance of the reflex phenomena in spastic paralysis of the lower limbs. Brain 1914;37:269-336.

${ }^{4}$ Walshe FMR. The Babinski plantar response, its forms, and its physiological and pathological significance. Brain 1956;79:529-56.

${ }^{5}$ Babinski MJ. Sur le réflexe cutané plantaire dans certaines affections organiques du systeme nerveux central. CR Soc Biol, (Paris) 1896;48:207-8.

6 Marie P, Foix Ch. Les réflexes d'automatism médullaire et le phénoméne des raccourcisseurs; leur valeur sémiologique leur signification physiologique. Revue Neurol (Paris) 1912;23:657-76.

${ }^{7}$ Brain WR, Wilkinson M. Observations on the extensor plantar reflex and its relationship to the functions of the pyramidal tract. Brain 1959;82:297-320.

${ }^{8}$ Landau WM, Clare MH. The plantar reflex in man, with special reference to some conditions where the extensor response is unexpectedly absent. Brain 1959;82:321-55.

${ }^{9}$ Kugelberg E, Eklund K, Grimby L. An electromyographic study of the nociceptive reflexes of the lower limb. Mechanism of the plantar response. Brain 1960;83:394-410.

${ }^{10}$ Tower SS. Pyramidal lesions in the monkey. Brain 1940;63:36-90.

$"$ Twitchell TE. The restoration of motor function following hemiplegia in man. Brain 1951;74:443-80.

12 Landau WM. The upper motor neuron syndrome. In: Neurological Pathophysiology. Eliasson SG, Pensky AL, Hardin WB, eds. Oxford University Press, pp. 117-132;1974.

13 Van Gijn J. B́abinski response: stimulus and effector. $J$ Neurol, Neurosurg Psychiatry 1975;38:180-6. 